\title{
Current Opinion on Pixantrone in the Treatment of Non-Hodgkin B-Cell Lymphoma
}

This article was published in the following Dove Press journal:

Therapeutics and Clinical Risk Management

\author{
Barbara Jezeršek Novaković (iD \\ Lučka Boltežar (10) \\ Aleksander Novaković (iD) \\ Division of Medical Oncology, Institute of \\ Oncology Ljubljana, Ljubljana, I000, \\ Slovenia
}

\begin{abstract}
Not many treatment options exist for patients with relapsed or refractory $(\mathrm{R} / \mathrm{R})$ B-cell non-Hodgkin lymphoma (NHL) in whom first- and second-line therapies were unsuccessful. This is especially true for patients with aggressive lymphomas. The innovative agent pixantrone has shown some promising results in terms of disease-free and overall survival, both in monotherapy as well as in combinations. However, recent trials (Phase III and realworld studies) reported unsatisfactory results, thereby raising the question about the role of pixantrone in the current treatment of R/R aggressive lymphomas. Nonetheless, there might still be a potential position for this drug in combinations, for use as first-line treatment of patients with cardiac dysfunction. This article summarizes the definition, structure, mechanism of action and reduced cardiotoxicity of pixantrone as well as efficacy and toxicity both in monotherapy and in combinations, as treatment for aggressive and indolent non-Hodgkin lymphomas.
\end{abstract}

Keywords: pixantrone, B-cell non-Hodgkin lymphomas, efficacy, toxicity

\section{Introduction}

First-line therapy in patients with aggressive lymphomas usually comprises an anthracycline-based regimen combined with rituximab, such as R-CHOP (rituximab, cyclophosphamide, doxorubicin, vincristine and prednisolone). However, only approximately $60-65 \%$ of patients can be cured with standard front-line therapy, while the remaining $35-40 \%$ of patients will exhibit primary refractory disease or relapse following an initial response to therapy. ${ }^{1-4}$ The overall outcome for these patients is quite poor with a chance of cure no more than $10 \%{ }^{2}$ The dismal outcomes for many patients who fail to respond to initial treatment, including patients refractory to potentially curative front-line treatment (median overall survival (OS) 7.1 months) or relapsing within 1 year of autologous stem cell transplantation (ASCT) (median OS 6.2 months), have been outlined in the SCHOLAR-1 analysis. $^{5}$

The standard second-line salvage therapy habitually includes a platinum and/or gemcitabine-based regimen, followed by consolidative high dose therapy and autologous stem cell transplantation (ASCT) in younger, fit patients. ${ }^{6,7}$ A recent real-world analysis discovered that only $33 \%$ of patients with $R / R$ diffuse large B-cell lymphoma (DLBCL) were planned for ASCT and finally, only $13 \%$ of patients actually underwent ASCT. $^{8}$ The median OS in patients after ASCT was 21.4 months, compared to 10.5 months in patients unfit for

\footnotetext{
Correspondence: Barbara Jezeršek Novaković

Division of Medical Oncology, Institute of Oncology Ljubljana, Zaloška 2, Ljubljana, 1000 , Slovenia

Tel $+386|587963|$

Fax +38615879305

Email bjezersek@onko-i.si
}

ASCT. ${ }^{8}$ 
Up until now, there has been no consensus regarding the third and further line therapies in multiply relapsed or refractory aggressive B-cell NHL. Typically, they consist of polychemotherapy combinations excluding the anthracyclines or of monotherapy with oxaliplatin, etoposide, gemcitabine, vinorelbine, etc. ${ }^{7,9}$ The first, and for quite some time the only, registered drug for multiply relapsed aggressive B-cell lymphomas was pixantrone. ${ }^{10,11}$ However, the phase III study PIX306, comparing the efficacy and safety of pixantrone with rituximab (PIX-R) versus gemcitabine with rituximab (GEM-R) in patients with relapsed (not refractory) aggressive B-cell NHL, deemed ineligible for ASCT (NTC01321541), failed to demonstrate any improvement in outcomes with pixantrone and rituximab as opposed to gemcitabine with rituximab. ${ }^{12-14}$ In line with this were also findings of some real-world studies ${ }^{15-17}$ which have shown overall disappointing outcomes of treatment with pixantrone and have therefore questioned the actual role of pixantrone in the current treatment of $\mathrm{R} / \mathrm{R}$ aggressive lymphomas.

Recently, further options were also offered to adult patients with relapsed or refractory diffuse large B-cell lymphomas. The first option is the chimeric antigen receptor T-cell (CAR T) therapy indicated after two or more lines of systemic therapy. This treatment option, though considerably effective, is associated with significant toxicities and represents an extensive financial burden to health-care systems. ${ }^{18-22}$

The second option - polatuzumab vedotin in combination with bendamustine and rituximab - has only very recently been approved for treatment of patients with relapsed/refractory DLBCL who are not candidates for hematopoietic stem cell transplant, ${ }^{23}$ however, it has not yet been reimbursed in quite a few EU countries.

Yet another treatment option for relapsed (not refractory) DLBCL, the anti-CD19 antibody tafasitamab in combination with lenalidomide, followed by tafasitamab monotherapy, has entered the formal regulatory review process in the EU in May 2020, on the basis of results of the Phase II L-MIND study. ${ }^{24}$

Lastly, in June 2020, selinexor (an oral selective inhibitor of nuclear export) received accelerated Food and Drug Administration (FDA) approval. The approval was based on the response rate observed in the phase IIb SADAL study for selinexor's second indication until now, which is treatment of adult patients with relapsed or refractory DLBCL after at least two lines of systemic therapy. The marketing authorization application for selinexor, as treatment for relapsed or refractory DLBCL, is planned for submission to the European Medicines Agency (EMA) in 2021. ${ }^{25}$

This review summarizes the definition, structure, mechanism of action and reduced cardiotoxicity of pixantrone, as well as efficacy and toxicity in monotherapy and in combinations in aggressive and indolent non-Hodgkin lymphomas and aims to identify the current role of pixantrone in the treatment of B-cell non-Hodgkin lymphoma.

\section{Pixantrone - Definition and Structure, Mechanism of Action, Mechanism of Reduced Cardiotoxicity}

Pixantrone is a novel aza-anthracenedione drug developed to reduce the likelihood of cardiotoxicity without compromising antineoplastic activity. ${ }^{26}$

The development of anthracyclines started in the 1950s (doxorubicin and daunorubicin), and was followed by the development of anthracenedione mitoxantrone in the 1970s. Anthracyclines block the function of topoisomerase II (isoforms $\alpha$ and $\beta$ ) - the enzyme that affects topologic features of DNA, resulting in the disruption of tumor cells. ${ }^{27}$ Unfortunately, anthracyclines also exhibit significant cardiotoxicity; presumably by driving reactions resulting in formation of free radicals and generation of reactive oxygen species, which in succession compromises the function of cells. ${ }^{27}$ As well as oxidative damage, topoisomerase II $\beta$ mediated responses to DNA damage may further contribute to anthracycline-induced cardiotoxicity. ${ }^{28}$

The structure of pixantrone differs from that of mitoxantrone in that the hydroquinone moiety was removed, a nitrogen heteroatom was inserted in the same ring and ethylamino-diethylamino side chains were substituted for hydroxyethylamino-ethylamino side chains. ${ }^{29,30}$ Pixantrone directly alkylates DNA, thereby forming stable DNA adducts and inducing DNA double-strand breaks, which prevents DNA replication, transcription and repair. ${ }^{10,26,28,31,32}$ Pixantrone also induces a latent type of DNA damage that impairs mitosis. ${ }^{33}$

In contrast to anthracyclines, pixantrone does not bind iron, indicating it has less potential to generate reactive oxygen species or form long-lasting alcohol metabolites. ${ }^{10,28-30}$ Pixantrone is also a relatively weak inhibitor of topoisomerase II paralleled to other anthracenediones and anthracyclines ${ }^{10,33}$ and, in contrast to mitoxantrone, more selectively inhibits topoisomerase II $\alpha$ versus 
topoisomerase II $\beta^{28}$ Pixantrone exhibited less cardiotoxic potential than mitoxantrone and doxorubicin in murine models. $^{34-36}$

\section{Effectiveness of Pixantrone in Monotherapy \\ Aggressive Lymphomas \\ First-Line Treatment}

To the best of our knowledge, there are no data on firstline treatment of aggressive lymphomas with pixantrone monotherapy.

\section{Treatment of $R / R$ Patients}

Overall, there were three Phase I studies which investigated the optimal dose and schedule of pixantrone - one in patients with NHL and chronic lymphocytic leukemia ${ }^{37}$ and two in patients with various solid tumors. ${ }^{38,39}$ The schedule recommended for phase II trials was $50 \mathrm{mg} / \mathrm{m}^{2}$ of active substance of pixantrone on days 1,8 and 15 of a 28-day cycle.

Borchmann et al evaluated pixantrone as a single agent in a phase II study in relapsed NHL patients who have received a median of two previous treatments. The overall response rate (ORR) was $27 \%$ with $15 \%$ of patients exhibiting complete response $(\mathrm{CR})$ and the median progression-free survival (PFS) was 3.5 months, while the overall survival (OS) was 7.6 months. $^{40}$

Pixantrone received conditional marketing approval by the European Medicines Agency for the treatment of multiply relapsed or refractory aggressive NHL on the basis of phase III trial PIX301. ${ }^{11}$ In this study, Pettengell et al compared the effectiveness of pixantrone in monotherapy against single-agent physicians' choice therapy (gemcitabine, mitoxantrone, etoposide, ifosfamide, oxaliplatin or vinorelbine) in the population of patients with aggressive $\mathrm{NHL}$, that were either refractory or relapsed after at least two prior regimens. Patients received $50 \mathrm{mg} / \mathrm{m}^{2}$ of active pixantrone on days 1,8 , and 15 of a 28 -day cycle. In the pixantrone group, $20 \%$ of patients achieved complete response or unconfirmed complete response $(\mathrm{CRu})$ versus $5.7 \%$ in the comparator group $(\mathrm{p}=0.021)$ and overall response rates of $37.1 \%$ versus $14.3 \% \quad(p=0.003)$. Furthermore, the median progression-free survival was significantly longer in the pixantrone arm (5.3 months versus 2.6 months, $\mathrm{p}=0.005$ ) with a trend towards a longer OS which was, however, not statistically significant (10.2 months versus 7.6 months, $\mathrm{p}=0.251) .{ }^{11}$ Posthoc analyses of this study demonstrated that neither previous therapy with rituximab nor the clinical response to last therapy influenced the outcome in the pixantrone arm. Additionally, a significantly higher $\mathrm{CR} / \mathrm{CRu}$ rate was observed in the subgroup of patients with centrally confirmed aggressive B-cell NHL who were receiving pixantrone, versus $\mathrm{CR} / \mathrm{CRu}$ rates observed in patients treated with a comparator agent as third- or fourth-line therapy. $^{41,42}$

Following the pivotal PIX301 study, there were two real-life studies on pixantrone in monotherapy; one UKwide retrospective study of R/R DLBCL patients ${ }^{15}$ and the other - the PIXA study conducted in Spain and Italy including the same patient population, that is, patients with aggressive relapsed or refractory B-cell NHL who had progressed after two or more prior therapies. ${ }^{16}$ Patients from the Eyre and coworkers' study had poorer prognostic features than those from the PIX301 trial; 85\% had refractory disease and $72 \%$ had an international prognostic index (IPI) value of 3 to 5 at commencement of pixantrone. The median PFS was 2.0 months and the median OS 3.4 months. The ORR was 24\% (CR 10\% and partial response (PR) 14\%) based on which the authors concluded that pixantrone had only limited activity in a cohort of high risk, predominantly refractory DLBCL patients. Multivariate Cox regression revealed that patients who relapsed more than 12 months after first-line treatment, those with fewer prior lines of therapy and relapsed (non-refractory) patients had better outcomes in terms of PFS. $^{15}$ Therefore, Eyre et al assumed, that the major population of unmet need are those with refractory DLBCL who are poorly represented within trials. ${ }^{15}$ Sancho et al reported that their study population had received a median of three prior therapies and, again, $84.6 \%$ were refractory to the last regimen. Median PFS was 2.8 months and median OS 4.0 months, with an ORR of 29\% (CR 13.2\% and PR 15.8\%). Patients receiving two or more cycles of pixantrone exhibited a slightly superior outcome (mPFS 3.1 and mOS 6.0 months, respectively, ORR $36.8 \%$ ). Even though the outcomes were numerically quite comparable to the outcomes of Eyre et al, Sancho et al concluded that pixantrone was effective in a realworld population of multiply $\mathrm{R} / \mathrm{R}$ patients with aggressive B-cell NHL, many of whom had very poor prognostic factors. ${ }^{16}$ This raises the question of definition, when can a certain drug be considered effective, as a median PFS of 2.8 months and a median OS of 4.0 months can hardly be considered as substantial evidence of effectiveness. 


\section{Indolent Lymphomas}

Up to our knowledge, there are no data on first or subsequent line treatments of patients with indolent lymphomas with pixantrone monotherapy.

\section{Effectiveness of Pixantrone in Combinations}

Aggressive Lymphomas

\section{First-Line Treatment}

The R-CPOP21 regimen (rituximab, pixantrone 150mg/ $\mathrm{m}^{2}$, cyclophosphamide, vincristine, prednisone) was investigated in an effort to minimize anthracycline-related cardiotoxicity in a comparative phase II study in adult patients with CD20+ DLBCL. ${ }^{43}$ Efficacy results showed, that the R-CPOP was an active regimen in this setting and the safety results showed substantially lower cardiotoxicity compared to R-CHOP. The $\mathrm{CR} / \mathrm{CRu}$ response rate was $75 \%$ with R-CPOP and $84 \%$ with R-CHOP. The study was underpowered to confirm the non-inferiority of $\mathrm{R}$-CPOP versus R-CHOP, due to premature termination of enrolment on account of regulatory constraints. Nonetheless, other efficacy endpoints were, for example: mPFS was not reached in the R-CPOP regimen, while it was 40 months in the R-CHOP arm. The authors concluded that, whereas non-inferiority was not demonstrated, the cardiotoxicity data seemed to support the pre-clinical characteristics of pixantrone, as compared with doxorubicin, and that their results supported further investigation of pixantrone in first-line therapy in high cardiac risk patients. ${ }^{43}$

Another ongoing phase II study is evaluating R-CPOP in elderly patients with DLBCL, including those with limited cardiac function (EudraCT number: 2014-005069-60). ${ }^{44}$ The study enrolls patients with DLBCL or follicular lymphoma grade IIIB in two prespecified populations: elderly patients $\geq 75$ years not eligible for standard R-CHOP21 and patients with impaired cardiac function ( $\mathrm{EF} \geq 40 \%$ and $\leq 50 \%$ ). Initial results, reported from one of the centers, for 10 patients, suggest, that this regimen is feasible and well tolerated with expected toxicities. ${ }^{45}$ All patients had clinical cardiac heart failure (CHF) at baseline and eight patients completed four to six cycles of R-CPOP, without experiencing higher-grade acute cardiac toxicity while on treatment. Complete remissions were observed in five patients $(50 \%)$ and current mOS was 10 months. ${ }^{45}$

\section{Treatment of R/R Patients}

Pixantrone with Rituximab

As the efficacy of pixantrone from the PIX301 study had yet to be confirmed in combination with rituximab to meet post-authorization requirements, the phase III study PIX306 was initiated. It was designed to compare the efficacy and safety of pixantrone with rituximab (PIX-R) versus gemcitabine with rituximab (GEM-R) in patients with relapsed (not refractory) aggressive B-cell NHL, deemed ineligible for ASCT (NTC01321541). ${ }^{12,13}$ The primary efficacy endpoint of PFS was not reached, as the median PFS for PIX+R group was 7.3 months versus 6.3 months in the GEM-R group (HR $0.85 ; \mathrm{p}=0.28$ ). The superior PFS of the control GEM-R arm (6.3 months) compared to the investigators' expectations for said control arm (2.8 months), was speculated to be due to the exclusion of patients with refractory DLBCL. Furthermore, the median OS for PIX-R of 13.3 months was worse than the median OS for GEM-R (19.6 months; HR 1.13). No patient subgroups could be identified for whom the PIX-R arm was statistically more beneficial in terms of survival. ${ }^{14}$

\section{CPOP}

Borchmann et al evaluated the potential efficacy of CPOP in a phase I/II study in adult patients $(n=35 / 30)$ with relapsed aggressive NHL who had previously received CHOP (with or without rituximab) and were deemed ineligible for $\mathrm{ASCT}^{46}{ }^{4}$ With this regimen, $\mathrm{CR} / \mathrm{CRu}$ rate of $47 \%$ and a median CR duration of 10.5 months were observed. The ORR was $73 \%$ and mOS was 17.9 months. The small study size precluded the authors from making definitive conclusions about cardiac safety and they noted that their patients already had a mean prior doxorubicinequivalent exposure of about $300 \mathrm{mg} / \mathrm{m}^{2}$ at baseline. They suggested that the frequency of clinically significant cardiac adverse events could be considered lower than expected if their patients had instead been treated with six cycles of $\mathrm{CHOP}^{46}$

\section{PREBEN/PEBEN}

It has been proposed that in $\mathrm{R} / \mathrm{R}$ aggressive NHL bendamustine and etoposide may be ideal for use in combination with pixantrone (PEBEN regimen) as they may act synergistically with pixantrone. ${ }^{29}$ Preliminary clinical experience with this combination (with rituximab in CD20+ lymphomas (PREBEN regimen)) was presented by d'Amore and coworkers at the ASH conference in 
$2014,{ }^{47}$ was extended by the same group in $2016^{48}$ and a Spanish group in $2017 .{ }^{49}$ These early results suggested that PREBEN/PEBEN is a feasible salvage regimen in patients with $\mathrm{R} / \mathrm{R}$ DLBCL, transformed indolent lymphoma and peripheral T-cell lymphoma, showing durable and substantial responses to treatment in individual patients. In thirty heavily pretreated patients, the ORR was $50 \%$ (complete metabolic response (CMR) 27\% and partial metabolic response (PMR) 23\%) with response duration of two to more than twenty-three months. ${ }^{48}$ Additionally, there were differences in response in the DLBCL subgroup between multiple-relapse patients and patients with refractory disease, as the response had been far worse in patients with primary refractory disease. ${ }^{48}$ PREBEN/PEBEN also acted as a successful bridging therapy to allogeneic transplant in some of the patients both in this preliminary clinical experience, ${ }^{48}$ as well as in the study of the Spanish group. ${ }^{49}$

On the basis of these encouraging results a phase I/II trial was initiated by the Nordic Lymphoma Group in collaboration with the HOVON group (EudraCT No. 2015-000758-39), ${ }^{50}$ investigating PREBEN/PEBEN as a salvage treatment for heavily pretreated patients with aggressive B or T-cell lymphomas. ${ }^{51,52}$ In phase I part of the study, the maximum tolerated doses of PREBEN were: pixantrone $50 \mathrm{mg} / \mathrm{m}^{2}$ day 1 and 8 , etoposide $100 \mathrm{mg} / \mathrm{m}^{2}$ day 1 , bendamustine $90 \mathrm{mg} / \mathrm{m}^{2}$ day 1 , and rituximab $375 \mathrm{mg} / \mathrm{m}^{2}$ day $1 .{ }^{51}$ Preliminary results, reported for 10 patients after two cycles of treatment, showed an ORR of $100 \%$ (40\% CMR and 60\% PMR). ${ }^{52}$

Polish Lymphoma Research Group's real-life analysis of PREBEN/PEBEN confirmed good efficacy of this regimen with 25 consecutive R/R NHL patients showing an ORR of $68 \%$ ( $40 \% \mathrm{CR}$ and $28 \%$ PR). At the median follow-up of 7.5 months, the mPFS and mOS were not reached; the projected PFS and OS at 12 months were $68 \%$ and 78\%, respectively. Quite important, however, was the observation that response rates were even higher in patients chemosensitive to their prior regimen (ORR $87.5 \%$; including $50 \% \mathrm{CR}$ ). ${ }^{53}$

Different in terms of efficacy were, however, the outcomes of another real-world study by Novakovic et al, reporting treatment results of twelve consecutive patients treated with pixantrone monotherapy (2 patients) or PREBEN/PEBEN ( $8 / 2$ patients, respectively). ${ }^{17}$ The prognostic features of these patients were far worse compared to the Polish analysis ${ }^{53}$ and the preliminary study of d'Amore, ${ }^{48}$ with $100 \%$ of patients having been refractory to last treatment and a high proportion of primary refractory disease, as well as insensitivity to primary anthracycline treatment by PIX301 criteria. In this analysis, the ORR was $0 \%$ and the median pixantrone specific OS was 3.5 months. The authors emphasized the importance of an appropriate selection of patients for this treatment, discouraging its use in patients with refractory disease. ${ }^{17}$

\section{PSHAP}

The PSHAP regimen (pixantrone $80 \mathrm{mg} / \mathrm{m}^{2}$ (base form dose) day 1 , methylprednisolone $500 \mathrm{mg}$ days 1 to 5 , cisplatin $25 \mathrm{mg} / \mathrm{m}^{2}$ days 1 to 4 , and cytarabine $2000 \mathrm{mg} /$ $\mathrm{m}^{2}$ day 5) was assessed for efficacy and toxicity in a phase I/II study in adult patients with $\mathrm{R} / \mathrm{R}$ aggressive NHL (mainly DLBCL), all of whom had received prior doxorubicin. ${ }^{54}$ Efficacy results were particularly promising after a median of four cycles (CR 37\%; PR 21\%; ORR $58 \%$ ) and the authors concluded that this regimen is an active salvage regimen which should be further evaluated as a pretransplant cytoreductive regimen. ${ }^{54}$

\section{Indolent Lymphomas}

\section{First-Line Treatment}

To the best of our knowledge, there are no data on firstline treatments of patients with indolent lymphomas with pixantrone in combinations.

\section{Treatment of R/R Patients}

\section{Pixantrone with Rituximab}

Though not approved for use in patients with $\mathrm{R} / \mathrm{R}$ indolent lymphomas, pixantrone has been evaluated in this setting (mostly follicular lymphomas grade I and II), combined with rituximab, in a phase III study. ${ }^{55}$ The results suggested that pixantrone and rituximab may be more effective than rituximab alone, giving an ORR of $75 \%$ versus $33 \%$, CR of $35 \%$ versus $11 \%$, PR of $40 \%$ versus $22 \%$ and time to progression of 13.2 versus 8.1 months. ${ }^{55}$

\section{FPD-R}

Pixantrone has also been evaluated in patients with relapsed indolent NHL (mostly follicular lymphoma and small lymphocytic lymphoma), combined with fludarabine, dexamethasone and rituximab (FDP-R), in a phase I study. ${ }^{56}$ This regimen was shown to be highly active with the ORR of $89 \%$, the CR of $63 \%$, uCR $7 \%$, and PR $19 \%$, as well as producing durable responses (median response twenty-three months). ${ }^{56}$ 


\section{Safety and Tolerability of Pixantrone}

Overall, pixantrone treatment seems to be safe and controllable. In various trials, there were no unexpected adverse events reported and no trials were closed prematurely because of side effects. In an assessment of twelve clinical trials with pixantrone the most common side effect of all grades was hematological toxicity, mainly neutropenia ( $50 \%$ of patients; grade $3 / 441 \%$ ), followed by anemia, leukopenia, and thrombocytopenia. Other side effects included asthenia, pyrexia and nausea, while some patients experienced reversible skin discoloration. ${ }^{57}$

In the pivotal PIX301 study monotherapy with pixantrone was mainly well tolerated. ${ }^{11}$ Adverse events were reported in $97 \%$ of patients receiving pixantrone with treatment-related adverse events occurring in $81 \%$ of patients. Adverse events of any grade ensuing in $\geq 10 \%$ of patients in the pixantrone arm were as follows: neutropenia in $50 \%$ of recipients, anemia in $31 \%$, leukopenia in $25 \%$, pyrexia in $24 \%$, asthenia in $24 \%$, cough in $22 \%$, thrombocytopenia in $21 \%$, decreased left ventricular ejection fraction (LVEF) in 19\%, nausea in $18 \%$, abdominal pain in $16 \%$, peripheral edema in $15 \%$, fatigue in $13 \%$, dyspnea in $13 \%$, alopecia in $13 \%$, constipation in $12 \%$, mucosal inflammation in $12 \%$, and skin discoloration in $10 \%$ of recipients. Serious adverse events were observed in $51 \%$ of patients receiving pixantrone and grade 3 or 4 adverse events occurred in $76 \%$ with neutropenia and leukopenia being the most common. ${ }^{11}$ Cardiac adverse events occurred in $35 \%$ of patients receiving pixantrone. An asymptomatic reduction of LVEF, with the median change of $-4 \%$ from baseline, was the most frequently observed cardiac adverse event. ${ }^{11}$ Only nine cases of cardiac events were considered related to pixantrone $(13 \%)$ and all were asymptomatic decreases of LVEF. ${ }^{58}$ There was no evidence of a cumulative, dose-related decline in ejection fraction in pixantrone recipients ${ }^{11}$ and no demonstrable relationship between the cumulative pixantrone dose and the manifestation of congestive heart failure. ${ }^{58}$ Death within 30 days of the last dose of pixantrone occurred in ten patients $(15 \%)$, but was related to progressive disease in five patients, while only one death of septic shock was considered related to pixantrone treatment. ${ }^{11}$

Various authors concluded that like with other cytotoxic chemotherapies, myelosuppression was quite common with pixantrone $^{11,59}$ and this remained the case when pixantrone was given in combination therapy. ${ }^{43,45,46,48,51,52,54,56}$ Overall, there appeared to be no new and unexpected adverse events with pixantrone in combination therapy (reviewed by Lekue et al). ${ }^{60}$ Evidence from the combination therapy trials reviewed by Lekue et al, ${ }^{60}$ as well as those from pixantrone monotherapy reviewed by Keating ${ }^{9}$ also tend to confirm an acceptable safety profile in terms of cardiotoxicity. Nevertheless, it remains important to continue to evaluate cardiotoxicity in all future trials. ${ }^{59}$ In addition to repeat LVEF assessment, determination of LV global longitudinal strain and follow-up of cardiac biomarkers (high sensitive-cardiac troponins (TnI or TnT), BNP or NT pro-BNP) could also be recommended at regular intervals. ${ }^{61}$

Studies specifically including patients with pre-existing cardiac dysfunction (eg, first-line treatment with CPOP) are of outmost significance. ${ }^{44,45}$ In a phase II trial, comparing R-CPOP with R-CHOP in untreated DLBCL, serious cardiac adverse events were more common in the R-CHOP arm ${ }^{43}$ and in the ongoing phase II study ${ }^{44}$ none of the eight evaluable patients with clinical CHF at baseline receiving R-CPOP experienced higher-grade cardiac toxicity. ${ }^{45}$

\section{Place of Pixantrone in the Treatment of Non-Hodgkin B-Cell Lymphoma}

As already mentioned in the introduction part, only few treatment options exist for patients with relapsed or refractory $(\mathrm{R} / \mathrm{R}) \mathrm{B}$-cell non-Hodgkin lymphoma (NHL) who fail first- and second-line therapies. The dismal outcomes have been outlined by the SCHOLAR-1 analysis for patients with refractory DLBCL who fail to respond to initial or subsequent salvage treatment. ${ }^{5}$ Even though certain relapsed patients with more indolent and lower risk disease might do relatively well with other palliative therapies, there is an urgent need for effective treatments for patients with refractory DLBCL whose disease fails to respond to immunochemotherapy or any subsequent salvage regimen and for those whose disease relapses early post-ASCT. ${ }^{5}$ The major population of unmet need are therefore patients with refractory DLBCL.

Of late, two treatments were approved for $R / R$ DLBCL: CAR-T-cell therapy and polatuzumab vedotin in combination with rituximab and bendamustine. Both treatments reported impressive results in clinical trials and are about to change the position of pixantrone in the setting of DLBCL $^{20,62,63}$ However, no real-world data are yet available for these two treatments and while at least the former is associated with significant toxicity, both represent 
a substantial financial burden. Recently, two further treatment options have either entered or are about to enter into the formal regulatory review process in EU, namely tafasitamab in combination with lenalidomide ${ }^{24}$ and selinexor. $^{25}$

On the other hand, for quite some time the only drug licensed for later lines of therapy in the R/R setting was pixantrone, approved on basis of PIX301 trial results. It has been proven efficacious while exhibiting acceptable toxicity in monotherapy, ${ }^{11,40-42}$ as well as in combinations, for $\mathrm{R} / \mathrm{R}$ aggressive lymphomas. ${ }^{46-49,52-54}$ Certain studies, ${ }^{15,17,48}$ however, have stated its lesser activity in refractory patients compared to relapsed ones and most of real-world analyses have shown inferior results compared to trial results, ostensibly at least partly on account of inclusion of patients with poor risk characteristics. ${ }^{15-17}$ Nevertheless, a good number of studies evaluated only clinical features of patients, with very few studies addressing the relation of histological characteristics of lymphomas to clinical outcome. ${ }^{17}$ In the study of Novakovic et al, it was observed that all patient samples were BCL-2 positive, predicting a higher probability of resistance to systemic treatment and a lesser OS. ${ }^{17}$ In this study, specifically, the ORR was $0 \%{ }^{17}$

Finally, the results of the phase III confirmatory study PIX306, comparing the efficacy of pixantrone with rituximab versus gemcitabine with rituximab in patients with relapsed (not refractory) aggressive B-cell NHL, failed to demonstrate an improvement in outcomes of treatment with pixantrone and rituximab against gemcitabine with rituximab. The primary efficacy endpoint of PFS was explicitly not reached and the median OS for pixantrone with rituximab of 13.3 months was worse than the median OS for gemcitabine with rituximab, which was 19.6 months. For none of the patient subgroups was the PIX$\mathrm{R}$ arm statistically more beneficial in terms of survival. ${ }^{14}$ These findings, together with the data from certain realworld studies, ${ }^{15-17}$ raised a serious concern about the actual role of pixantrone in the R/R DLBCL setting, where it has been primarily approved. Regarding the emerging new treatment options mentioned above, ${ }^{20,24,25,62,63}$ as well as some others cited it the BJH commentary by Eyre, ${ }^{64}$ it is very likely that pixantrone will no longer be a part of the solution to the unmet need of patients with refractory (and relapsed) DLBCL.

Despite that, potentially, pixantrone could still remain the treatment option for a very select population of patients: patients with relapsed (not refractory) aggressive lymphoma (DLBCL), otherwise ineligible for high dose treatment or CAR T-cell therapy, primarily sensitive to anthracyclines according to PIX301 criteria, whose disease is not primary refractory, with a tumor that is not BCL-2 positive, who have received fewer lines of therapy (not more than three), who have not received an excessive dose of anthracyclines in prior treatments and who do not have a high IPI score at commencement of pixantrone treatment. Unfortunately, there are not many such patients in the real-world setting.

Furthermore, there seem to exist two putative "niches" for use of pixantrone - one being the first-line treatment of aggressive lymphomas in patients with cardiac impairment and the second being the treatment of patients with $R / R$ indolent lymphomas.

Lately, the attention has mostly moved to whether pixantrone combined with cyclophosphamide, vincristine, prednisone, and rituximab may be a new effective and safe option in the first-line setting for aggressive NHL, specifically in patients with cardiac impairment, which is being explored in the phase II study, conducted at the University of Freiburg. ${ }^{44}$ Preliminary results suggest that this regimen is feasible and well tolerated. ${ }^{45}$ Herbrecht et $\mathrm{al}^{43}$ in their phase II study have already indicated that cardiotoxicity was lesser with R-CPOP than with R-CHOP, even though the noninferiority of R-CPOP was not demonstrated. Further research is certainly required prior to this indication for pixantrone becoming widely accepted, particularly since promising results have already been reported for the R-GCVP regimen (gemcitabine as a direct substitute for anthracycline), ${ }^{65}$ R-COMP regimen (liposomal doxorubicin as substitute for standard doxorubicin) ${ }^{66}$ and R-GEMOX regimen (gemcitabine combined with oxaliplatin and rituximab $)^{67}$ in these patients with cardiac impairment.

Although not approved for use in patients with $R / R$ indolent lymphomas, pixantrone has been shown to be highly active and well tolerated in the FDP-R regimen ${ }^{56}$ and in combination with rituximab. ${ }^{55}$ Nevertheless, the data of pixantrone in this setting are not mature and further research will be needed to unequivocally recommend the FDP-R regimen for patients with $\mathrm{R} / \mathrm{R}$ follicular lymphoma.

\section{Conclusion}

In line with the recent results of the PIX306 trial, where pixantrone and rituximab did not demonstrate improvement in outcomes, and with outcomes of several real-world studies there is no unequivocal role of pixantrone in the current treatment of $\mathrm{R} / \mathrm{R}$ aggressive lymphomas. 
It may remain a treatment option in a very select population, that is, in patients with relapsed (not refractory) aggressive lymphoma, who are ineligible for high dose treatment or CAR T-cell therapy, while being primarily sensitive to anthracyclines, have a tumor that is not BCL-2 positive, have received no more than three lines of therapy or an excessive dose of anthracyclines in prior treatments and do not have a high IPI score at commencement of pixantrone treatment.

Apart from that, pixantrone may prove useful in the first-line setting for aggressive NHL in patients with cardiac impairment and in patients with $\mathrm{R} / \mathrm{R}$ indolent lymphomas, however, further studies need to be performed before this becomes a standard of care.

\section{Author Contributions}

All authors made a significant contribution to the work reported, whether that is in the conception, study design, execution, acquisition of data, analysis and interpretation, or in all these areas; took part in drafting, revising or critically reviewing the article; gave final approval of the version to be published; have agreed on the journal to which the article has been submitted and agree to be accountable for all aspects of the work.

\section{Funding}

There is no funding to report.

\section{Disclosure}

BJN received speaker's honoraria from Janssen (Johnson \& Johnson), Roche, Servier and Takeda. The authors report no other conflicts of interest in this work.

\section{References}

1. Sarkozy C, Sehn LH. New drugs for the management of relapsed or refractory diffuse large B-cell lymphoma. Ann Lymphoma. 2019;3:10. doi: 10.21037/aol.2019.09.01

2. Friedberg JW. Relapsed/refractory diffuse large B-cell lymphoma. Hematol Am Soc Hematol Educ Progr. 2011;2011:498-505. doi:10.1182/asheducation-2011.1.498

3. Gregoric B, Zadnik V, Jezersek Novakovic B. The diffuse large B-cell lymphoma - where do we stand now in everyday clinical practice. Radiol Oncol. 2012;46(2):153-159. doi:10.2478/v10019-012-0002-6

4. Horvat M, Zadnik V, Južnič Šetina $T$, et al. Diffuse large B-cell lymphoma: 10 years' real-world clinical experience with rituximab plus cyclophosphamide, doxorubicin, vincristine and prednisolone. Oncol Lett. 2018;15(3):3602-3609. doi:10.3892/ol.2018.7774

5. Crump M, Neelapu SS, Farooq U, et al. Outcomes in refractory diffuse large B-cell lymphoma: results from the international SCHOLAR-1 study. Blood. 2017;130(16):1800-1808. doi:10.1182/blood-2017-03769620
6. Philip T, Guglielmi C, Hagenbeek A, et al. Autologous bone marrow transplantation as compared with salvage chemotherapy in relapses of chemotherapy-sensitive non-Hodgkin's lymphoma. $N$ Engl $J$ Med. 1995;333(23):1540-1545. doi:10.1056/NEJM199512073332305

7. Tilly H, Gomes da Silva M, Vitolo U, et al. Diffuse large B-cell lymphoma (DLBCL): ESMO clinical practice guidelines for diagnosis, treatment and follow-up. Ann Oncol. 2015;26(Supp15):V116V125. doi:10.1093/annonc/mdv304

8. Nabhan C, Klink A, Lee CH, Laney JR, Yang Y, Purdum AG. Overall survival (OS) and transplantation (ASCT) utilization in real-world patients with relapsed/refractory diffuse large B-cell lymphoma (RR-DLBCL) [ASCO abstract]. J Clin Oncol. 2018;36(15 Suppl 1):7545. doi:10.1200/JCO.2018.36.15_suppl.7545

9. Keating GM. Pixantrone: a review in relapsed or refractory aggressive non-hodgkin's lymphoma. Drugs. 2016;76(16):1579-1586. doi:10.1007/s40265-016-0650-8

10. European Medicines Agency. Pixuvri (pixantrone dimaleate): summary of product characteristics [document on the Internet]; 2016. Available from: https:/www.ema.europa.eu/en/documents/product-information /pixuvri-epar-product-information_en.pdf. Accessed October 10, 2020.

11. Pettengell R, Coiffier B, Narayanan G, et al. Pixantrone dimaleate versus other chemotherapeutic agents as a single-agent salvage treatment in patients with relapsed or refractory aggressive non-Hodgkin lymphoma: a Phase 3, multicentre, open-label, randomised trial. Lancet Oncol. 2012;13(7):696-706. doi:10.1016/S1470-2045(12)70212-7

12. Belada D, Georgiev P, Dakhil S, et al. Pixantrone-rituximab versus gemcitabine-rituximab in relapsed/refractory aggressive non-Hodgkin lymphoma. Future Oncol. 2016;12(15):1759-1768. doi:10.2217/fon2016-0137

13. Salles GA, Jurczak W, Andorsky DJ, et al. Results of a phase 3 randomised multicenter study comparing pixantrone + rituximab with gemcitabine + rituximab in patients with relapsed aggressive B-cell non-hodgkin lymphoma not eligible for stem cell transplantation [ASH abstract]. Blood. 2018;132(Suppl1):4189. doi:10.1182/blood2018-99-112455

14. Pettengell R, Długosz-Danecka M, Andorsky D, et al. Pixantrone plus rituximab versus gemcitabine plus rituximab in patients with relapsed aggressive B-cell non-Hodgkin lymphoma not eligible for stem cell transplantation: a phase 3, randomized, multicentre trial (PIX306). $\mathrm{Br}$ J Haematol. 2020;188(2):240-248. doi:10.1111/bjh.16255

15. Eyre TA, Linton KM, Rohman P, et al. Results of a multicentre UK-wide retrospective study evaluating the efficacy of pixantrone in relapsed, refractory diffuse large B cell lymphoma. Br J Haematol. 2016;173(6):896-904. doi:10.1111/bjh.14021

16. Sancho J-M, Navarro B, Soler Campos JA, et al. Efficacy and safety of pixantrone for the treatment of multiply relapsed or refractory aggressive non-Hodgkin B-cell lymphomas. Eur $J$ Haematol. 2020;104(5):499-508. doi:10.1111/ejh.13392

17. Novakovic A, Boltezar L, Jezersek novakovic B. Limited efficacy of pixantrone in refractory diffuse large B-cell lymphoma. Oncol Lett. 2020;19(3):2028-2034. doi:10.3892/ol.2020.11288

18. Schuster SJ, Svoboda J, Chong EA, et al. Chimeric antigen receptor T cells in refractory B-cell lymphomas. $N$ Engl $J$ Med. 2017;377 (26):2545-2554. doi:10.1056/NEJMoa1708566

19. Schuster SJ, Bishop MR, Tam CS, et al. Tisagenlecleucel in adult relapsed or refractory diffuse large B-cell lymphoma. $N$ Engl J Med. 2019;380(1):45-56. doi:10.1056/NEJMoa1804980

20. Neelapu SS, Locke FL, Bartlett NL, et al. Axicabtagene ciloleucel CAR T-cell therapy in refractory large B-cell lymphoma. $N$ Engl J Med. 2017;377(26):2531-2544. doi:10.1056/NEJMoa1707447

21. Neelapu SS, Tummala S, Kebriaei P, et al. Chimeric antigen receptor T-cell therapy - assessment and management of toxicities. Nat Rev Clin Oncol. 2018;15(1):47-62. doi:10.1038/nrclinonc.2017.148

22. Hernandez I, Prasad V, Gellad WF. Total costs of chimeric antigen receptor T-cell immunotherapy. JAMA Oncol. 2018;4(7):994-996. doi:10.1001/jamaoncol.2018.0977 
23. Sehn LH, Herrera AF, Flowers CR, et al. Polatuzumab vedotin in relapsed or refractory diffuse large B-cell lymphoma. J Clin Oncol. 2020;38(2):155-165. doi:10.1200/JCO.19.00172

24. Maddocks KJ, Duell J, González Barca E, et al. Update of the single-arm phase II L-MIND study of MOR208 + lenalidomide (LEN) in relapsed/refractory diffuse large B-cell lymphoma (R-R DLBCL): response rates in patient subgroups with poor prognosis [ASCO abstract]. J Clin Oncol. 2019;37(15Suppl 1):7521. doi:10.1200/JCO.2019.37.15_suppl.7521

25. Karyopharm Therapeutics Inc.. Karyopharm announces FDA approval of XPOVIO $^{\circledR}$ (selinexor) for the treatment of patients with relapsed or refractory diffuse large B-cell lymphoma (DLBCL) [press release on the Internet]; 2020. Available from: https://investors.karyopharm.com/202006-22-Karyopharm-Announces-FDA-Approval-of-XPOVIO -R-selinexor-for-the-Treatment-of-Patients-with-Relapsed-or-RefractoryDiffuse-Large-B-cell-Lymphoma-DLBCL. Accessed January 22, 2021.

26. Volpetti S, Zaja F, Fanin R. Pixantrone for the treatment of adult patients with relapsed or refractory aggressive non-Hodgkin B-cell lymphomas. Onco Targets Ther. 2014;7:865-872. doi:10.2147/OTT. S 34055

27. Sawyer DB. Anthracyclines and heart failure. $N$ Engl $J$ Med. 2013;368(12):1154-1156. doi:10.1056/NEJMcibr1214975

28. Hasinoff BB, Wu X, Patel D, Kanagasabai R, Karmahapatra S, Yalowich JC. Mechanisms of action and reduced cardiotoxicity of pixantrone; a topoisomerase II targeting agent with cellular selectivity for the topoisomerase II $\alpha$ isoform. $J$ Pharmacol Exp Ther. 2016;356(2):397-409. doi:10.1124/jpet.115.228650

29. Menna P, Salvatorelli E, Minotti G. Rethinking drugs from chemistry to therapeutic opportunities: pixantrone beyond anthracyclines. Chem Res Toxicol. 2016;29(8):1270-1278. doi:10.1021/acs.chemrestox.6b00190

30. Salvatorelli E, Menna P, Paz OG, et al. The novel anthracenedione, pixantrone, lacks redox activity and inhibits doxorubicinol formation in human myocardium: insight to explain the cardiac safety of pixantrone in doxorubicin-treated patients. $J$ Pharmacol Exp Ther. 2013;344(2):467-478. doi:10.1124/jpet.112.200568

31. Pettengell R, Kaur J. Pixantrone dimaleate for treating non-Hodgkin's lymphoma. Expert Opin Orphan Drugs. 2015;3(6):747-757. doi:10.1517/21678707.2015.1042454

32. Evison BJ, Mansour OC, Menta E, Phillips DR, Cutts SM. Pixantrone can be activated by formaldehyde to generate a potent DNA adduct forming agent. Nucleic Acids Res. 2007;35 (11):3581-3589. doi:10.1093/nar/gkm285

33. Beeharry N, Di Rora AGL, Smith MR, Yen TJ. Pixantrone induces cell death through mitotic perturbations and subsequent aberrant cell divisions. Cancer Biol Ther. 2015;16(9):1397-1406. doi:10.1080/ 15384047.2015.1070979

34. Beggiolin G, Crippa L, Menta E, et al. Bbr 2778, an aza-anthracenedione endowed with preclinical anticancer activity and lack of delayed cardiotoxicity. Tumori. 2001;87(6):407-416. doi: $10.1177 / 030089160108700611$

35. Cavalletti E, Crippa L, Mainardi P, et al. Pixantrone (BBR 2778) has reduced cardiotoxic potential in mice pretreated with doxorubicin: comparative studies against doxorubicin and mitoxantrone. Invest New Drugs. 2007;25(3):187-195. doi:10.1007/s10637-007-9037-8

36. Longo M, Della Torre P, Allievi C, Morisetti A, Al-Fayoumi S, Singer JW. Tolerability and toxicological profile of pixantrone $\left(\right.$ Pixuvri $\left.^{\circledR}\right)$ in juvenile mice. Comparative study with doxorubicin. Reprod Toxicol. 2014;46:20-30. doi:10.1016/j.reprotox.2014.02.006

37. Borchmann P, Schnell R, Knippertz R, et al. Phase I study of BBR 2778 , a new aza-anthracenedione, in advanced or refractory nonHodgkin's lymphoma. Ann Oncol off J Eur Soc Med Oncol. 2001;12(5):661-667. doi:10.1023/a:1011139016294

38. Faivre S, Raymond E, Boige V, et al. A phase I and pharmacokinetic study of the novel aza-anthracenedione compound BBR 2778 in patients with advanced solid malignancies. Clin Cancer Res. 2001;7 (1):43-50.
39. Dawson LK, Jodrell DI, Bowman A, et al. A clinical phase I and pharmacokinetic study of BBR 2778, a novel anthracenedione analogue, administered intravenously, 3 weekly. Eur J Cancer. 2000;36 (18):2353-2359. doi:10.1016/s0959-8049(00)00342-7

40. Borchmann P, Morschhauser F, Parry A, et al. Phase-II study of the new aza-anthracenedione, BBR 2778, in patients with relapsed aggressive nonHodgkin's lymphomas. Haematologica. 2003;88(8):888-894.

41. Pettengell R, Sebban C, Zinzani PL, et al. Monotherapy with pixantrone in histologically confirmed relapsed or refractory aggressive B-cell non-Hodgkin lymphoma: post-hoc analyses from a phase III trial. Br J Haematol. 2016;174(5):692-699. doi:10.1111/bjh.14101

42. Pettengell R, Coiffier B, Egorov A, Singer J, Sivcheva L. Long-term response and remission with pixantrone in patients with relapsed or refractory aggressive non-hodgkin lymphoma: post-hoc analysis of the multicenter, Open-label, randomized PIX301 trial. Clin Drug Investig. 2018;38(6):527-533. doi:10.1007/s40261-018-0635-3

43. Herbrecht R, Cernohous $P$, Engert A, et al. Comparison of pixantrone-based regimen (CPOP-R) with doxorubicin-based therapy (CHOP-R) for treatment of diffuse large B-cell lymphoma. Ann Oncol off J Eur Soc Med Oncol. 2013;24(10):2618-2623. doi:10.1093/annonc/ mdt 289

44. University of Freiburg. R-CPOP as first-line therapy for elderly patients with DLBCL and for patients with limited cardiac function with DLBCL. Available from: https://www.clinicaltrialsregister.eu/. EudraCT number: 2014-005069-60. Accessed October 10, 2020.

45. Marks R, Strüßmann T, Krohn U, Duyster J, Finke J, Fritsch K. Feasibility of pixantrone containing R-CPOP as first-line treatment for patients with aggressive B cell lymphoma with congestive heart failure or at risk of anthracycline induced cardiotoxicity [EHA abstract]. HemaSphere. 2018;2(Suppl 1):813.

46. Borchmann P, Herbrecht R, Wilhelm M, et al. Phase I/II study of pixantrone in combination with cyclophosphamide, vincristine, and prednisone in patients with relapsed aggressive non-Hodgkin lymphoma. Leuk Lymphoma. 2011;52(4):620-628. doi:10.3109/10428194.2010.546016

47. d'Amore F, Joergensen J, Sillesen IB, et al. Preliminary clinical experience on the efficacy and feasibility of a new combination regimen consisting of pixantrone, etoposide, and bendamustine with or without the addition of rituximab in patients with relapsed/refractory aggressive non-Hodgkin lymphomas [ASH abstract]. Blood. 2014;124(8):5435. doi:10.1182/blood.V124.21.5435.5435

48. Clausen MR, Leppa S, Brown PN, et al. The combination of pixantrone, etoposide, bendamustine and, in $\mathrm{CD} 20+$ tumors, rituximab (PREBEN) shows promising feasibility/efficacy in heavily pretreated aggressive lymphomas of B- and T-cell phenotype - results of the pre-trial experience leading to a Nordic Phase 1/2 study (the PREBEN trial) [ASH abstract]. Blood. 2016;128(22):1782. doi:10.1182/blood.V128.22.1782.1782

49. Barrenetxea Lekue C, Alonso Caballero C, Hernández Vázquez L, et al. Preben: pixantrone, rituximab, etoposide and bendamustine, Spanish experience in diffuse large B-cell lymphoma treatment [ASH abstract]. Blood. 2017;130(Suppl 1):5235.

50. Aarhus University Hospital. Phase $1 / 2$ study of the combination of pixantrone, etoposide, bendamustine and, in CD20 positive tumors, rituximab in patients with relapsed aggressive non-hodgkin lymphomas of B- or T-cell phenotype - the P[R]EBEN study. Available from: https://www.clinicaltrialsregister.eu/. EudraCT No. 2015-000758-39. Accessed October 10, 2020.

51. Jørgensen J, Leppä S, Relander T, et al. A phase Ib, open label, multicenter trial of pixantrone, etoposide, bendamustine and, in CD20-positive tumors, rituximab (PREBEN) in relapsed aggressive lymphomas of Bor T-cell phenotype [EHA abstract]. HemaSphere. 2018;2(Suppl 1):808.

52. d'Amore F, Leppä S, Larsen TS, et al. A phase 1/2 study of pixantrone, etoposide, bendamustine and, in $\mathrm{CD} 20+$ tumors, rituximab in patients with relapsed aggressive B- or T-cell lymphomas - the P[R] EBEN study [ICML abstract]. Hematol Oncol. 2017;35 (Suppl2):423-424. doi:10.1002/hon.2440_7 
53. Długosz-Danecka M, Hus I, Puła B, et al. Pixantrone, etoposide, bendamustine, rituximab $(\mathrm{P}[\mathrm{R}] \mathrm{EBEN})$ as an effective salvage regimen for relapsed/refractory aggressive non-Hodgkin lymphoma-Polish Lymphoma Research Group real-life analysis. Pharmacol Rep. 2019;71 (3):473-477. doi:10.1016/j.pharep.2019.02.001

54. Lim S-T, Fayad L, Tulpule A, et al. A phase I/II trial of pixantrone (BBR2778), methylprednisolone, cisplatin, and cytosine arabinoside (PSHAP) in relapsed/refractory aggressive non-Hodgkin's lymphoma. Leuk Lymphoma. 2007;48(2):374-380. doi:10.1080/ 10428190601060496

55. Santoro A, Voglova J, Gabrail N, et al. Comparative trial of BBR 2778 (pixantrone) + rituximab vs single agent rituximab in the treatment of relapsed/refractory indolent non-Hodgkin's lymphoma (NHL) [ASCO abstract]. J Clin Oncol. 2006;24(18Suppl 1):7578. doi:10.1200/jco.2006.24.18_suppl.7578

56. Srokowski TP, Liebmann JE, Modiano MR, et al. Pixantrone dimaleate in combination with fludarabine, dexamethasone, and rituximab in patients with relapsed or refractory indolent non-Hodgkin lymphoma: phase 1 study with a dose-expansion cohort. Cancer. 2011;117 (22):5067-5073. doi:10.1002/cncr.26121

57. Boyle EM, Morschhauser F. Pixantrone: a novel anthracycline-like drug for the treatment of non-Hodgkin lymphoma. Expert Opin Drug Saf. 2015;14(4):601-607. doi:10.1517/14740338.2015.1010505

58. European Medicines Agency. Pixuvri (pixantrone): CHMP assessment report [document on the Internet]. London: Committee for Medicinal Products for Human Use (CHMP); 2012. Available from: https://www.ema.europa.eu/en/documents/assessment-report/pixuvriepar-public-assessment-report_en.pdf. Accessed October 10, 2020.

59. Papadatos-Pastos D, Pettengell R. Pixantrone: merging safety with efficacy. Expert Rev Hematol. 2013;6(1):25-33. doi:10.1586/ehm.12.61

60. Barrenetxea Lekue C, Grasso Cicala S, Leppä S, et al. Pixantrone beyond monotherapy: a review. Ann Hematol. 2019;98 (9):2025-2033. doi:10.1007/s00277-019-03749-0
61. Curigliano G, Lenihan D, Fradley M, et al. Management of cardiac disease in cancer patients throughout oncological treatment: ESMO consensus recommendations. Ann Oncol off J Eur Soc Med Oncol. 2020;31(2):171-190. doi:10.1016/j.annonc.2019.10.023

62. Borchmann P, Tam CS, Jäger U, et al. An updated analysis of JULIET, a global pivotal Phase 2 trial of tisagenlecleucel in adult patients with relapsed or refractory diffuse large B-cell lymphoma [EHA abstract]. HemaSphere. 2018;2(Suppl 1):347.

63. Morschhauser F, Flinn IW, Advani R, et al. Polatuzumab vedotin or pinatuzumab vedotin plus rituximab in patients with relapsed or refractory non-Hodgkin lymphoma: final results from a phase 2 randomised study (ROMULUS). Lancet Haematol. 2019;6(5):e254e265. doi:10.1016/S2352-3026(19)30026-2

64. Eyre TA. Another disappointment in treating relapsed, refractory high-risk aggressive B-cell lymphomas. Br J Haematol. 2020;188 (2):202-204. doi:10.1111/bjh.16259

65. Fields PA, Townsend W, Webb A, et al. De novo treatment of diffuse large B-cell lymphoma with rituximab, cyclophosphamide, vincristine, gemcitabine, and prednisolone in patients with cardiac comorbidity: a United Kingdom National Cancer Research Institute trial. J Clin Oncol. 2014;32(4):282-287. doi:10.1200/JCO.2013.49.7586

66. Luminari S, Viel E, Ferreri AJM, et al. Nonpegylated liposomal doxorubicin combination regimen in patients with diffuse large B-cell lymphoma and cardiac comorbidity. Results of the HEART01 phase II trial conducted by the Fondazione Italiana Linfomi. Hematol Oncol. 2018;36(1):68-75. doi:10.1002/hon.2425

67. Shen Q-D, Zhu H-Y, Wang L, et al. Gemcitabine-oxaliplatin plus rituximab (R-GemOx) as first-line treatment in elderly patients with diffuse large B-cell lymphoma: a single-arm, open-label, phase 2 trial. Lancet Haematol. 2018;5(6):e261-e269. doi:10.1016/S23523026(18)30054-1
Therapeutics and Clinical Risk Management

\section{Publish your work in this journal}

Therapeutics and Clinical Risk Management is an international, peerreviewed journal of clinical therapeutics and risk management, focusing on concise rapid reporting of clinical studies in all therapeutic areas, outcomes, safety, and programs for the effective, safe, and sustained use of medicines. This journal is indexed on PubMed Central, CAS,

\section{Dovepress}

EMBase, Scopus and the Elsevier Bibliographic databases. The manuscript management system is completely online and includes a very quick and fair peer-review system, which is all easy to use. Visit http://www.dovepress.com/testimonials.php to read real quotes from published authors. 